\title{
Can management of the components of metabolic syndrome modify the course of chronic kidney disease?
}

\author{
Yaeni Kim ${ }^{(\mathbb{D}}$, Cheol Whee Park \\ Division of Nephrology, Department of Internal Medicine, Institute for Aging and Metabolic Diseases, College of Medicine, The \\ Catholic University of Korea, Seoul, Republic of Korea
}

Metabolic syndrome (MetS), described for the first time by Reaven in 1988, refers to a set of conditions including central obesity, systemic hypertension, hyperglycemia, and dyslipidemia [1]. The criteria for MetS have changed since its original description. The concept of insulin resistance that leads to subsequent chronic inflammation and endothelial dysfunction lies at its core [2]. Chronic kidney disease (CKD) has become a significant public health burden affecting a considerable proportion of both the adult and elderly populations. Diabetes and hypertension are established causal and triggering factors that contribute to development and progression of CKD. Evidence demonstrates that an imbalance in lipid metabolism and its accumulation in ectopic tissues exerts lipotoxicity damaging the kidneys [2]. Alterations in lipid profiles in the state of positive energy balance that promotes hypertrophy and hyperplasia of white adipose tissues of visceral fat may also play a significant role in the pathogenesis of CKD [3]. There exists a widely accepted notion that MetS is a major risk factor for the de-

\footnotetext{
Received May 4, 2020; Revised May 18, 2020;

Accepted May 19, 2020

Edited by Gheun-Ho Kim, Hanyang University, Seoul, Republic of Korea
}

Correspondence: Cheol Whee Park

Division of Nephrology, Department of Internal Medicine, Seoul St. Mary's Hospital, College of Medicine, The Catholic University of Korea, 222 Banpo-daero, Seocho-gu, Seoul 06591, Republic of Korea.E-mail: cheolwhee@hanmail.net

Copyright (c) 2020 by The Korean Society of Nephrology

(a) This is an open-access article distributed under the terms of the Creative Commons Attribution Non-Commercial License (http://creativecommons. org/licenses/by-nc-nd/4.0/), which permits unrestricted non-commercial use, distribution, and reproduction in any medium, provided the original work is properly cited. velopment of type 2 diabetes and cardiovascular disease. The relationship between MetS and its contribution to renal impairment has recently been validated in largescale, population-based studies $[3,4]$. It has been demonstrated that MetS is an independent risk factor for CKD. The more severe are the components comprising the syndrome, the higher is the risk for CKD development (Fig. 1). Therefore, it is inferable that the presence of these comorbidities would identify subjects at increased risk for type 2 diabetes, cardiovascular disease, and allcause mortality. Furthermore, the observed association between MetS and risk for renal dysfunction raises the question as to whether correcting one or more of the characteristics of MetS may effectively prevent or deter renal functional deterioration.

In this issue of Kidney Research and Clinical Practice, Park et al [5] substantiated the association between CKD risk factors and development of MetS. A distinguishing feature of the current study is that it further explored the impact of alterations in metabolic status on CKD development by analyzing whether recovery from preexisting MetS was related to a decreased risk for CKD, and vice versa. The authors demonstrated that the MetSdeveloped group had a lower risk of CKD compared to the MetS-chronic group, and those who recovered from preexisting MetS had a higher risk for CKD than those initially free of MetS. This suggests that the chronicity and precedent burden of MetS may be individualized, may exert a deleterious impact on kidneys, and may not fully resolve in a few years.

When unraveling the pathophysiology of MetS that predisposes patients to CKD (Fig. 1), insulin resistance is responsible for a chain reaction associated with defec- 


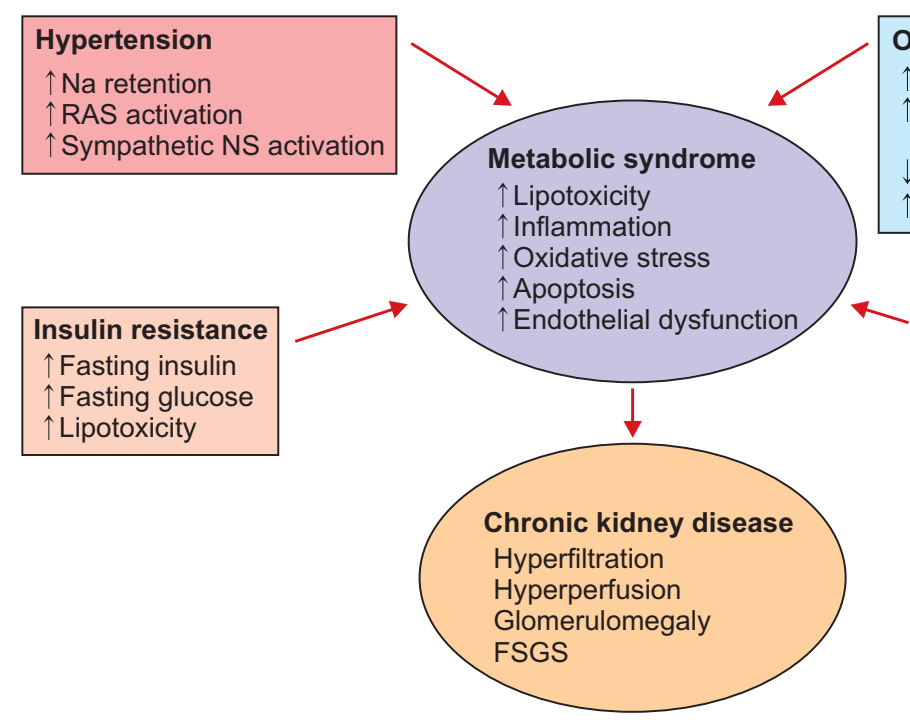

tive lipid metabolism in the peripheral tissues including skeletal muscle, adipose tissue, and the liver. Defective glucose uptake in the skeletal muscle leads to chronic hyperinsulinemia, inadequate lipolytic effect of insulin in adipose tissues, and impaired ability to suppress glucose production in the liver. This comprises the underlying mechanism that activates the signaling pathways associated with de novo lipogenesis and altered lipid metabolism. As a result, increased plasma free fatty acid production and its accumulation in the target organ inflict direct lipotoxicity in association with decreased endothelial production of nitric oxide. This cascade increases oxidative stress, inflammation, and apoptosis, which have been implicated in progression of diabetic kidney disease [6]. An altered lipid profile in MetS is characterized by increase in triglycerides and low-density-lipoprotein cholesterol and decrease in high-density-lipoprotein cholesterol concentrations. It is expected that targeting this lipid profile to within the lower limit of the normal range may deter CKD progression in association with decreased inflammation and oxidative stress in endothelial cells [7]. The mechanism of obesity-induced renal damage is considered to result from a combination of hemodynamic and metabolic abnormalities. Both the glomerular filtration rate and renal plasma flow rise with obesity and diminished insulin response increases efferent arteriolar pressure resulting in glomerular hyperfiltration and hypertrophy. Renal ultrastructural findings associated with obesity often manifests as focal segmental glomerulosclerosis characterized by glomerulomegaly, podocytopathy, and mesangial

\section{Obesity}

$\uparrow \mathrm{BMI}, \uparrow \mathrm{WHR}$

$\uparrow$ Intraabdominal/

retroperitoneal pressure

$\downarrow$ Adiponectin, $\uparrow$ leptin

$\uparrow$ IL-6, $\uparrow$ TNF- $\alpha$

Dyslipidemia

$\uparrow T G, \uparrow$ LDL-C, $\downarrow$ HDL-C

$\uparrow$ IL-6, $\uparrow$ TGF- $\beta$
Figure 1. Contribution of the components of metabolic syndrome to chronic kidney disease development.

BMI, body mass index; FSGS, focal segmental glomerulosclerosis; HDL-C, highdensity-lipoprotein cholesterol; IL-6, interleukin-6; LDL-C, low-density-lipoprotein cholesterol; TG, triglycerides; TGF- $\beta$, transforming growth factor- $\beta$; TNF- $\alpha$, tumor necrosis factor $\alpha$; NS, nervous system; RAS, renin angiotensin system; WHR, waist hip ratio.

expansion presenting with massive proteinuria. An increase in certain circulatory cytokines such as leptin and adiponectin and inflammatory markers such as interleukin-6 and tumor necrosis factor $\alpha$ directly affects endothelial cells and podocytes in the kidneys [8]. Moreover, fat accumulation in and around the kidneys drives the above-mentioned processes and contributes to deterioration of CKD through increased renal arterial resistance and decline in glomerular filtration rate and hyperuricemia [9]. Enhanced sodium reabsorption and loss of a vasodilatory effect in the setting of hyperinsulinemia may lead to sodium retention and salt-sensitive high blood pressure, rendering one much more vulnerable to CKD development. Activation of this renin-angiotensin system may further deteriorate renal function in association with insulin resistance and hyperglycemia. The development of hypertension contributes to increased intraglomerular pressure and resultant injury to the epithelial lining, leading to microalbuminuria. The role of hypertension as the key player in the MetS and renal disease association was demonstrated in a previous study. Controlling hypertension eliminates the statistical association between MetS and renal disease [10]. This is in parallel with the current study in which hypertension development was associated with the most substantial increase in CKD risk [5].

Until now, there has been no clear-cut margin as to which of these individual mechanisms contributes to the risk of CKD development. Due to the complexity of their interrelationships, it is difficult to draw a causal associa- 
tion and discriminate which of the components of MetS gives rise to renal functional deterioration. Given the epidemiologic nature of this study, it is not proven whether the therapeutic interventions that aim to correct the various abnormalities of MetS can prevent the development and/or progression of renal damage. This study is noteworthy in that it strengthens and provides evidence for the role assigned to public metabolic risk management for prevention of CKD. Repetitive assessments of the status of MetS and identification of those at risk for CKD development will provide evidence for further interventional studies. These studies must aim to verify whether treating the components of MetS may confer a preventive outcome for the progression of CKD.

\section{Conflicts of interest}

All authors have no conflicts of interest to declare.

\section{Funding}

This study was supported by grants from the Basic Science Research Program through the National Research Foundation of Korea (NRF), funded by the Ministry of Education, Science, and Technology (YK; 2019R1I1A1A01059638, CWP; 2016R1A2B2015980, 2019R1H1A2079787).

\section{Authors' contributions}

Cheol Whee Park participated in the study design, coordination. Yaeni Kim wrote the manuscript. All authors read and approved the final manuscript.

\section{References}

[1] Reaven GM. Banting lecture 1988. Role of insulin resistance in human disease. Diabetes 1988;37:1595-1607.

[2] Locatelli F, Pozzoni P, Del Vecchio L. Renal manifestations in the metabolic syndrome. J Am Soc Nephrol 2006;17(4 Suppl 2):S81-S85.

[3] Huh JH, Yadav D, Kim JS, et al. An association of metabolic syndrome and chronic kidney disease from a 10-year prospective cohort study. Metabolism 2017;67:54-61.

[4] Hu Y, Shi LX, Zhang Q, Peng NC. Increased risk of chronic kidney diseases in patients with metabolic syndrome: a 3-year prospective cohort study. Curr Med Sci 2019;39: 204-210.

[5] Park S, Lee S, Kim Y, et al. Reduced risk for chronic kidney disease after recovery from metabolic syndrome: a nationwide population-based study. Kidney Res Clin Pract 2020; 39:180-191.

[6] Sarafidis PA, Ruilope LM. Insulin resistance, hyperinsulinemia, and renal injury: mechanisms and implications. Am J Nephrol 2006;26:232-244.

[7] Fried LF, Orchard TJ, Kasiske BL. Effect of lipid reduction on the progression of renal disease: a meta-analysis. Kidney Int 2001;59:260-269.

[8] Mende CW, Einhorn D. Fatty kidney disease: a new renal and endocrine clinical entity? Describing the role of the kidney in obesity, metabolic syndrome, and type 2 diabetes. Endocr Pract 2019;25:854-858.

[9] Lamacchia O, Nicastro V, Camarchio D, et al. Para- and perirenal fat thickness is an independent predictor of chronic kidney disease, increased renal resistance index and hyperuricaemia in type-2 diabetic patients. Nephrol Dial Transplant 2011;26:892-898.

[10] Thomas G, Sehgal AR, Kashyap SR, Srinivas TR, Kirwan JP, Navaneethan SD. Metabolic syndrome and kidney disease: a systematic review and meta-analysis. Clin J Am Soc Nephrol 2011;6:2364-2373. 